\title{
Neuropathogenic and non-neuropathogenic EHV-1 strains induce the accumulation of hyperphosphorylated Tau in primary murine neurons
}

\author{
J. CYMERYS' ${ }^{1}$ A. SŁOŃSKA ${ }^{1}$, M. CHODKOWSKI ${ }^{1}$, A. GOLKE ${ }^{1}$, M. KRZYŻOWSKA², M. W. BAŃBURA ${ }^{1}$
}

\begin{abstract}
${ }^{1}$ Department of Preclinical Sciences, Faculty of Veterinary Medicine, Warsaw University of Life Sciences, Warsaw, Poland; ${ }^{2}$ Military Institute of Hygiene and Epidemiology, Kozielska 4, 01-163, Warsaw, Poland
\end{abstract}

Received October 1, 2019; revised February 28, 2020; accepted March 11, 2020

\begin{abstract}
Summary. - Equid herpesvirus 1 (EHV-1) causes respiratory disease, abortion and neurological disorders in horses. Similarly, to other alphaherpesviruses, EHV-1 is neurotropic and establishes latency in the neurons of its natural host. Despite the fact that many studies have been devoted to the pathogenesis of various clinical forms of EHV-1 infection, mechanisms of the neuronal damage are still not fully understood. The aim of this study was to define the phosphorylation status of tau protein in neuronal cell culture infected with EHV-1. Phosphorylation of tau was tested at tau-ser199/ser202, tau-ser404, tau-ser262, tau-thr181, tau-thr217 and tau-thr205 sites. We described, for the first time, that EHV-1 infection leads to the accumulation of hyperphosphorylated tau in primary murine neurons. We showed that non-neuropathogenic and neuropathogenic EHV-1 strains specifically induce hyperphosphorylation of tau-ser199/ser202, tau-ser404 and tau-thr205 during long-term infection and after a controlled activation of productive infection.
\end{abstract}

Keywords: tau protein; hyperphosphorylation; equid herpesvirus 1 (EHV-1); neuronal cell culture

\section{Introduction}

Equid herpesvirus type 1 (EHV-1) is a worldwide pathogen of horses. Clinical signs of the disease are usually observed in young, immunologically unexperienced individuals being infected with EHV-1 for the first time. The infection can manifest by three clinical forms: respiratory, as rhinopneumonitis, neurological and by abortions. Equid herpesvirus myeloencephalopathy (EHM) is characterized by neurological symptoms varying from mild ataxia to enforced recumbency (Hussey et al., 2013). Despite the fact that many studies have been

E-mail: jcymerys@op.pl; phone: +4822-593-6055.

Abbreviations: $\mathrm{ACV}=$ acyclovir; $\mathrm{dpi}=\operatorname{day}(\mathrm{s})$ post infection; EHV-1 = equid herpesvirus 1; EHV-126 = EHV-1 neuropathogenic strain; Jan-E = EHV-1 non-neuropathogenic strain; hpi = hour(s) post infection; Tau = microtubule-associated protein; $\mathrm{TSA}=$ trichostatin A devoted to the pathogenesis of various clinical forms of EHV-1 infection, mechanisms of the neuronal damage are still not fully understood. What is especially true in reference to tau protein expression and phosphorylation, that it is the major microtubule-associated protein in the brain. Tau proteins are abundant in neurons, where they stabilize microtubules and are also one of the hallmarks of Alzheimer disease (Brunden et al., 2012; Himmelstein et al., 2012). There are few reports on human herpesvirus type 1 and type 2 (HHV-1 and HHV-2) infections, confirming the role of tau protein in neurodegenerative lesions (Kristen et al., 2015; Wozniak et al., 2009; Lin et al., 2002). Tau proteins are crucial in regulating the rate of microtubule polymerization. They also influence regulation of the axon diameter, axonal transport and neurogenesis. HHV-1 and HHV-2 infections modify tau proteins, mostly through phosphorylation, leading eventually to the formation of improperly folded, defective tau protein, which was recognized as a major component of neurofibrillary plaques, 
characteristic to taupathies, the neurodegenerative disorders (Alvarez et al., 2012; Kristen et al., 2015; Laurent et al., 2018). Over forty phosphorylation sites, activated by serine and threonine kinases have been identified/ recognized (Arendt et al., 2016). Thus, natively unfolded tau proteins stabilize microtubules and start to fold improperly to aggregate and lose their sensitivity to cellular proteinases. Formation of aggregates is a highly energy consuming process and it affects cellular membrane(s) permeability along with neuronal cell metabolism, leading to the toxic injury and cell death (Himmelstein et al., 2012; Arendt et al., 2016). Tau aggregation is probably common to all alphaherpesviruses.

Our previous studies had provided answers to a number of key EHV-1-related questions. We found out that non-neuropathogenic and neuropathogenic EHV-1 strains are able to replicate without the need for adaptation in murine neurons in vitro. Positive real-time PCR (qPCR) and nested PCR ( $\mathrm{NPCR}$ ) results indicated a significant increase of the DNA copy number of analyzed EHV-1 strains (up to 24 hours post infection; hpi), in comparison to the uninfected control (Cymerys et al., 2010, 2016, 2018). Moreover, using real-time cell growth analysis, we have demonstrated that primary murine neurons are able to survive long-term EHV-1 infection (3 weeks) (Cymerys et al., 2018). In spite of that, the question of interactions between the EHV-1 and neuronal cells is still pending, and further studies are necessary, so here we decided to investigate the phosphorylation state of tau protein in neuronal cell culture infected with EHV-1 in vitro.

\section{Materials and Methods}

Nerve cell cultures. Balb/c (H-2d) mice were used to establish primary culture of murine neurons, as described previously (Cymerys et al., 2010). Cells were plated onto coverslips and plates coated with poly-D-lysine/laminin ( $10^{4}$ neurons per well). Neuronal cultures were cultured in B-27 Neuron plating medium consisting of neurobasal medium, B27 supplement, glutamine (200 mmol/l), glutamate $(10 \mathrm{mmol} / \mathrm{l})$, antibiotics (penicillin and streptomycin, $1 \%$ ) with $10 \%$ supplement of fetal bovine (5\%) and equine serum (5\%) (Thermo Fisher Scientific). To eliminate non-neural cells, cultures were maintained in growth medium supplemented with $10 \mu \mathrm{M}$ cytosine $\beta$-D-arabinofuranoside (after 3 days for 24 hours) (Sigma-Aldrich). Next, the medium was removed and replaced with Neuron feeding medium (B-27 Neuron plating medium without glutamate). In this medium, murine neurons were maintained for next 6 days prior to treatments, at $37^{\circ} \mathrm{C}$ with $5 \% \mathrm{CO}_{2}$.

Viruses and inoculation of cells. Cultured neurons were infected with either of the two different neurotropic strains of EHV-1: (i) non-neuropathogenic strain Jan-E isolated from aborted fetus (lack of neuropathogenicity as confirmed by PCRRFLP neuropathogenic/non-neuropathogenic discrimination test) (Cymerys et al., 2016), from the collection of the Virology Laboratory at Warsaw University of Life Sciences-SGGW and (ii) neuropathogenic strain of EHV-1(EHV-126) isolated from aborted fetus in Hungary in 2004 (neuropathogenicity confirmed by PriProET technique) (Malik et al., 2010). Neurons were infected with Jan-E or EHV-1 26 strains of EHV-1 (MOI 1.0) for 60 min at $37^{\circ} \mathrm{C}$. After incubation, the inoculum was removed and fresh culture medium was added. Subsequently, infected cells were incubated for 1,2 , and 7 days at $37^{\circ} \mathrm{C}$ with $5 \% \mathrm{CO}_{2}$. Simultaneously, neurons were infected in the presence of acyclovir (ACV; $128 \mu \mathrm{g} / \mathrm{ml}$ ) for 7 days. Additionally, in the cell culture which has been infected in the presence of ACV, the culture medium was removed after 7 days of incubation and replaced with a fresh one without ACV, but with the addition of trichostatin A (TSA; 100 $\mu \mathrm{M})$, for another 7 days. The ACV concentration that effectively inhibits EHV-1 replication was established previously (Golke et al., 2017). Trichostatin A was used to stimulate productive EHV-1 infection, after ACV removal, as it is a known chemical reactivator of HHV-1 (Liu and Li, 2015). The replication kinetics of both EHV-1 strains in primary murine neurons and influence of ACV and TSA was determined previously (Golke et al., 2017).

Immunofluorescence analysis of Tau phosphorylation. Neurons seeded on glass coverslips in 12-well plates were infected with EHV-1. At 1 dpi (day post infection) cells were stained for detection of tau protein phosphorylation. At the beginning cells were washed twice in PBS and fixed in 3.7\% paraformaldehyde/ PBS (Sigma-Aldrich) for $30 \mathrm{~min}$ at room temperature (RT), then permeabilized with $0.5 \%$ TritonX-100 solution in PBS. Before staining, fixed cells were blocked with PBS containing $1 \%$ bovine serum albumin (BSA) for $30 \mathrm{~min}$ at room temperature. The cells were treated with Tau monoclonal antibody (Tau 5; total tau) or phospho-Tau Ser199/Ser202, phospho-Tau Ser404, phospho-Tau Ser262, phospho-Tau Thr181, phospho-Tau Thr217 and phospho-Tau Thr205 antibodies diluted in 1\% BSA (1:1000; Thermo Fisher Scientific) and incubated for $1 \mathrm{~h}$ at RT and then labeled with anti-mouse Texas Red and anti-rabbit IgG-Rhodamine RedX secondary antibody diluted in blocking solution (1:1000; Thermo Fisher Scientific) for $1 \mathrm{~h}$ at RT. The presence of viral antigen was detected by means of direct immunofluorescence, using polyclonal antiserum EHV-1/ERV conjugated to FITC (VMRD, Inc.). Cell nuclei were stained with Hoechst 33258, according to the manufacturer's recommendations. Next, coverslips were mounted on microscope slides using anti-fade mounting medium (Sigma-Aldrich). Confocal images were acquired using Fluoview FV10i laser scanning confocal microscope (Olympus Polska Sp. z o.o.) with a 10x air lens and 60x water-immersion lens, using Ultraviolet/Visible light LD lasers with excitation at $405 \mathrm{~nm}, 473 \mathrm{~nm}$ and $559 \mathrm{~nm}$ to excite Hoechst, FITC and TRITC, respectively. Images were captured using FV10i software (Olympus Polska Sp. z o.o.) and converted to 24-bit tiff files for visualization. 
Western blot analysis. Primary neuronal cells, prepared as described previously, were first washed with ice-cold PBS and lysed in N-PER Neuronal protein extraction reagent (Thermo Fisher Scientific) containing protease and phosphatase inhibitors (Halt phosphatase inhibitor cocktail and Halt protease inhibitor cocktail; Thermo Fisher Scientific), $20 \mathrm{~min}$ on ice. The lysates were clarified by centrifugation for $15 \mathrm{~min}$ at $4^{\circ} \mathrm{C}$. Quantification of the protein content in lysates was performed with Micro BCA protein assay kit (Thermo Fisher Scientific) and spectrophotometry on an Epoch BioTek spectrophotometer. Samples containing $20 \mu \mathrm{g}$ of proteins were incubated with Laemmli sample buffer containing $\beta$-ME (Bio-Rad, USA) for $5 \mathrm{~min}$ at $95^{\circ} \mathrm{C}$. Subsequently, the samples and protein markers were electrophoresed on a $10 \%$ polyacrylamide Bis-Tris plus gel with MES running buffer and transferred onto a PVDF membrane (Thermo Fisher Scientific). The membrane was blocked with 5\% BSA in TBST and incubated overnight with primary antibodies: total tau (Tau 5 mAb), phospho-Tau Ser199/Ser202, phospho-Tau Ser404, phospho-Tau Ser262, phospho-Tau Thr181, phospho-Tau Thr217 and phospho-Tau Thr205 (dilution 1:1000; Thermo Fisher Scientific). After several washes in $0.1 \%$ Tris-buffered saline (TBS)-Tween 20, membranes were incubated with HRP-conjugated secondary antibodies for $1 \mathrm{~h}$ at RT and developed using enhanced chemiluminescence (Clarity western ECL substrate; Bio-Rad). The protein bands were visualized by ChemiDoc ${ }^{\mathrm{TM}} \mathrm{MP}$ Imaging system (Bio-Rad, USA). Glyceraldehyde 3-phosphate dehydrogenase (GAPDH) was used as a loading control and for protein normalization during densitometry measurements. For densitometry analysis ImageLab 6.0.1 (BioRad) software was used.

Statistical analysis. Data were analyzed with GraphPad InStat version 3 (GraphPad Software Inc., USA). Data analyzed for statistical significance were subjected to a two-way analysis of variance (ANOVA). All results shown represent means and standard deviation (SD) of three independent experiments. Results with $p$-value $\leq 0.05$ were considered statistically significant.

\section{Results and Discussion}

Immunofluorescence analysis was used to determine whether EHV-1 infection modifies the phosphorylation state of tau in cultured neurons. For this purpose, anti-tau antibody (Tau 5), which reacts with a phosphorylationindependent epitope and phosphorylation-sensitive antibodies, anti-phospho-Tau: ser199/ser202, ser404, ser262, thr205, thr217 and thr181 were used. Figure 1a depicts the staining of control and EHV-1 infected neurons with anti-tau Tau 5 antibody demonstrating that tau protein was concentrated evenly on the surface of the neurite, axons and often found on the dendrites. Next, we measured total tau (Tau 5) protein expression in uninfected (a)
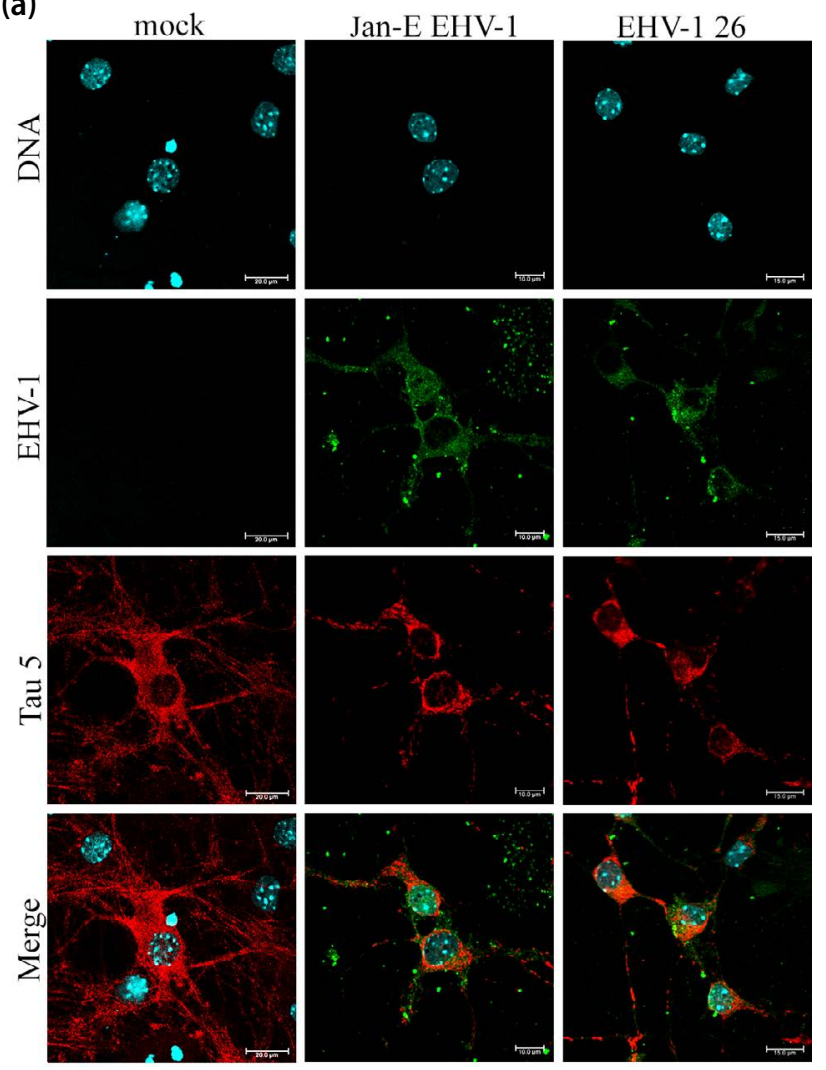

(b)

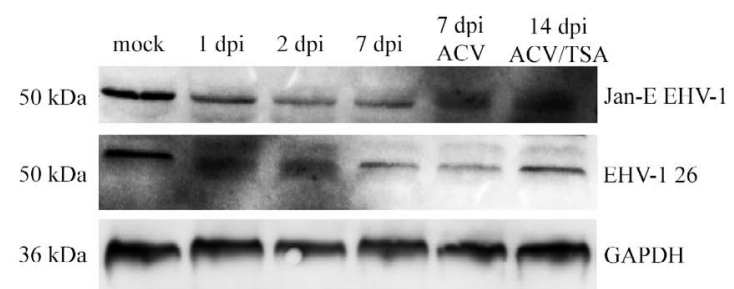

(c)

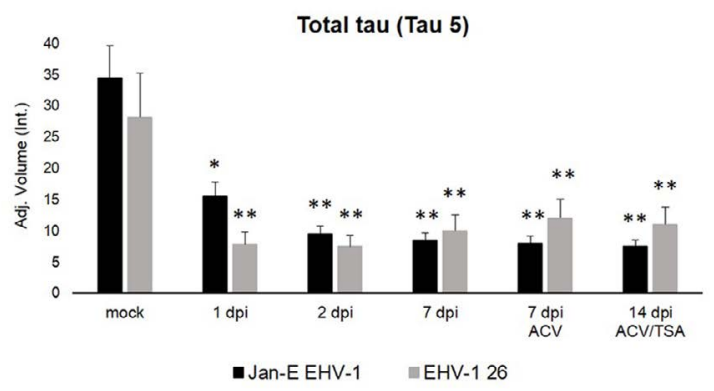

Fig. 1

Analysis of total Tau 5 protein expression

(a) Fluorescence images of mock-or EHV-1-infected primary murine neurons (1 dpi). Tau 5 is shown in red, EHV-1 antigens - green, DNA blue. (b) Western blot detection of Tau 5 in EHV-1-infected neurons at 1, 2 and $7 \mathrm{dpi}$. Acyclovir (ACV) was used to inhibit EHV-1 replication at $7 \mathrm{dpi}$. Trichostatin A (TSA) was used to stimulate productive EHV-1 infection at 14 dpi. GAPDH represents a loading control. (c) Densitometric analysis of Tau 5 protein levels normalized to GAPDH level in mock-infected and EHV-1-infected neurons; results were statistically compared to mock infected control $\left({ }^{*} \mathrm{p} \leq 0.05 ;{ }^{* *} \mathrm{p} \leq 0.01\right)$. 
(a)
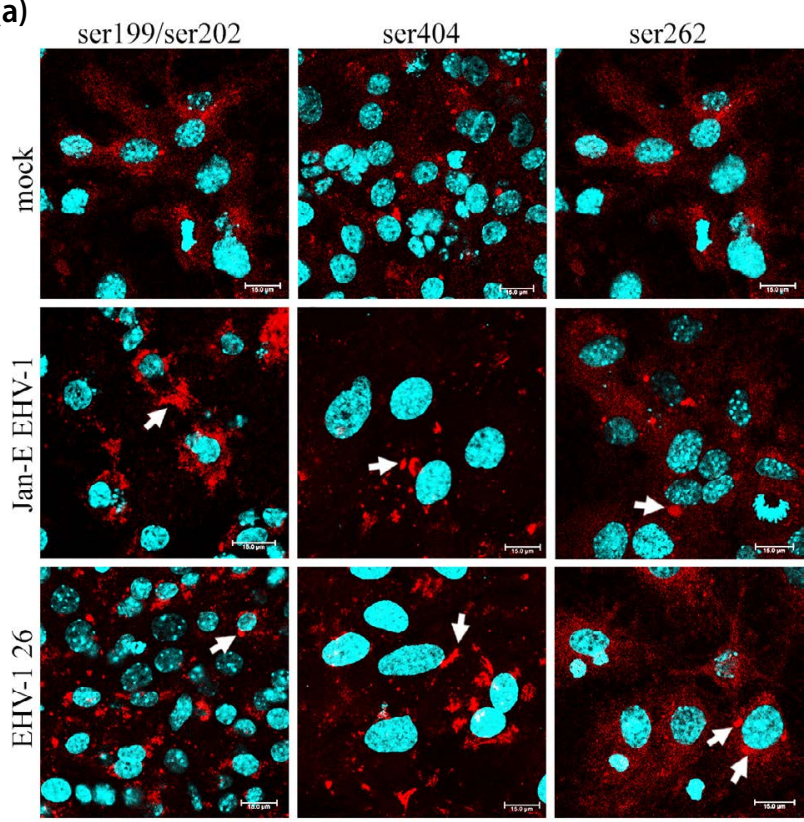

(b)

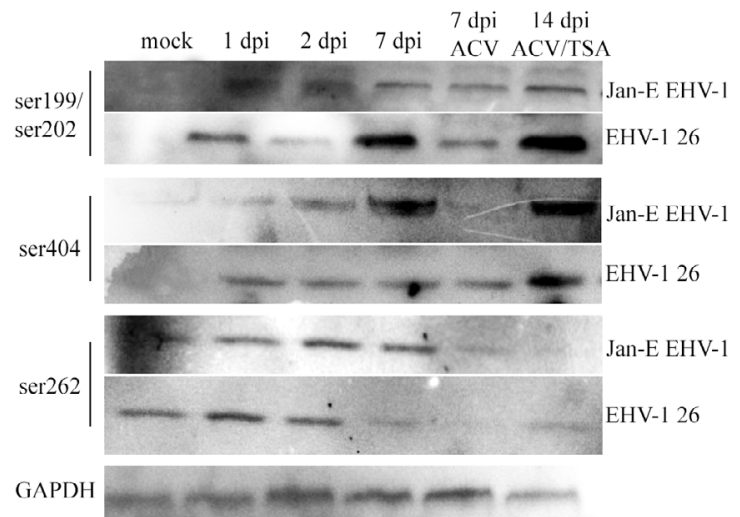

(c)

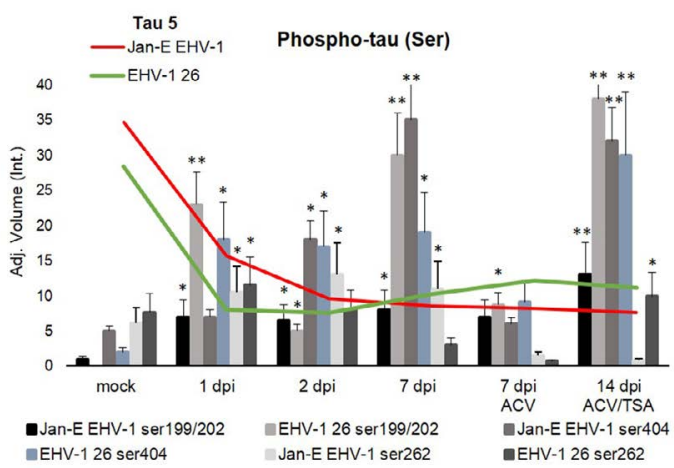

and infected neurons by Western blot and we observed reduction in the expression levels of this protein during infection in comparison to control samples (Fig. 1b). Tau protein expression was at the same level, regardless of the time after infection. The expression levels also did not change after inhibition of EHV-1 replication by ACV, as well as after a controlled activation of EHV-1 productive infection by TSA (Fig. 1c).

In uninfected neurons fluorescence intensity of tauphosphorylated epitopes was weak and detected mainly in the perinuclear area. When the cells were exposed to both tested strains of EHV-1 ( $24 \mathrm{hpi}$ ), we observed an accumulation of almost all epitopes of phosphorylated tau (Fig. 2a, 3a white arrows). The largest accumulation of phosphorylated tau, mainly in the cytoplasm around the nucleus was observed for the following epitopes: ser199/ ser202, ser404, ser262 and thr181. The phosphorylation of tau thr217 was poorly detectable, both in control and after EHV-1 infection (Fig. 2a, 3a). Interestingly, both Jan-E and EHV-1 26 strains induced the intranuclear accumulation of phospho-tau thr205, at viral replication sites (Fig. 3a yellow arrows), which was not observed in the case of other tau phosphorylations.

Three independent experiments of Western blot showed an increase in the phosphorylation level of tau ser199/ser202, ser404 and ser262 at 1, 2 and 7 dpi. A statistically significant increase in level of expression was demonstrated for ser199/202 after infection with neuropathogenic strain EHV-1 26 and ser404 after infection with both strains: Jan-E and EHV-1 26, compared to the control (Fig. 2 c). In the case of phosho-tau thr, a statistically significant increase was shown for thr205 after infection with Jan-E strain and EHV-126, compared to the control (Fig. 3c).

In addition, the level of tau protein phosphorylation changes was studied in neuronal cultures in which productive infection of EHV-1 was completely suppressed and activated again in controlled conditions. For this purpose, neurons were infected with neuropathogenic and non-neuropathogenic EHV-1 strains in the presence of antiviral drug ACV to suppress productive infection. Trichostatin A (TSA) was used to stimulate productive

Fig. 2

Analysis of tau phosphorylation (ser199/202, ser404, ser262) expression

(a) Fluorescent images of mock- or EHV-1 infected primary murine neurons (1 dpi). Phospho-tau is shown in red and DNA in blue. White arrows indicate accumulation of phosphorylated tau epitopes: ser199/202, ser404, ser262 around the nucleus. (b) Western blot detection of ser199/202, ser404 and ser262 in mock- and EHV-1- infected neurons. Acyclovir (ACV) was used to inhibit EHV-1 replication 7 dpi. Trichostatin A (TSA) was used to stimulate productive infection at 14 dpi. GAPDH represents a loading control. (c) Densitometric analysis of ser199/202, ser404 and ser262 protein levels (from three independent experiments) normalized to GAPDH level in mock-infected and EHV-1-infected neurons; results were statistically compared to mock infected control ( $\left.{ }^{*} \mathrm{p} \leq 0.05 ;{ }^{* *} \mathrm{p} \leq 0.01\right)$. Red (Jan-E EHV-1) and green (EHV-26 strain) lines present total Tau 5 level in EHV-1- infected neurons. 
infection, after ACV removal, in order to check whether hyperphosphorylation of tau was specifically provoked by EHV-1 infection.

The results obtained after these experiments confirmed that tau phosphorylation in neurons was specifically induced by EHV-1 infection. After controlled EHV-1 reactivation (both EHV-1 strains), a statistically significant increase of expression level of tau ser199/ser202, ser404 and thr205 was observed, both compared to the control and to the total Tau 5 expression (Fig. 2c, 3c red and green lines).

Hyperphosphorylation is believed to be an early event in the pathway that leads from soluble to insoluble and filamentous tau protein (Wozniak et al., 2009; Alvarez et al., 2012). The accumulation of hyperphosphorylated tau in the cell body of a neuron could contribute to the generation of PHFs - paired helical filaments - as a result of aggregation of abnormally phosphorylated tau protein, and consequently, formation of neurofibrillary tangles (NFTs). The appearance and accumulation of PHFs are clearly disease related - tau aggregation is considered to be toxic to the neurons in which it occurs (Himmelstein et al., 2012). Alvarez et al. (2012), in their cellular model demonstrated that HHV-1 infection provokes a specific increase in hyperphosphorylated tau in clearly defined nuclear regions corresponding to the compartments where replication and transcription of the viral DNA takes place. Tau hyperphosphorylation induced by HHV-1 may thus determine the recruitment of tau to the nucleus or may alter tau activity to promote the viral replication processes in neuronal cells, with the consequence of neurodegeneration.

In summary, the present findings indicate that the phosphorylation state and the intracellular distribution of tau are strongly affected in EHV-1 infected neuronal cells. Therefore, tau hyperphosphorylation induced by EHV-1 may contribute to the neuronal damage observed, so far, in neurodegenerative processes linked to HHV-1 infection. We can presume that tau hyperphosphorylation induced by EHV-1 may alter tau activity to promote the viral replication in neuronal cells. The functional significance of these differential phosphorylation events (a)
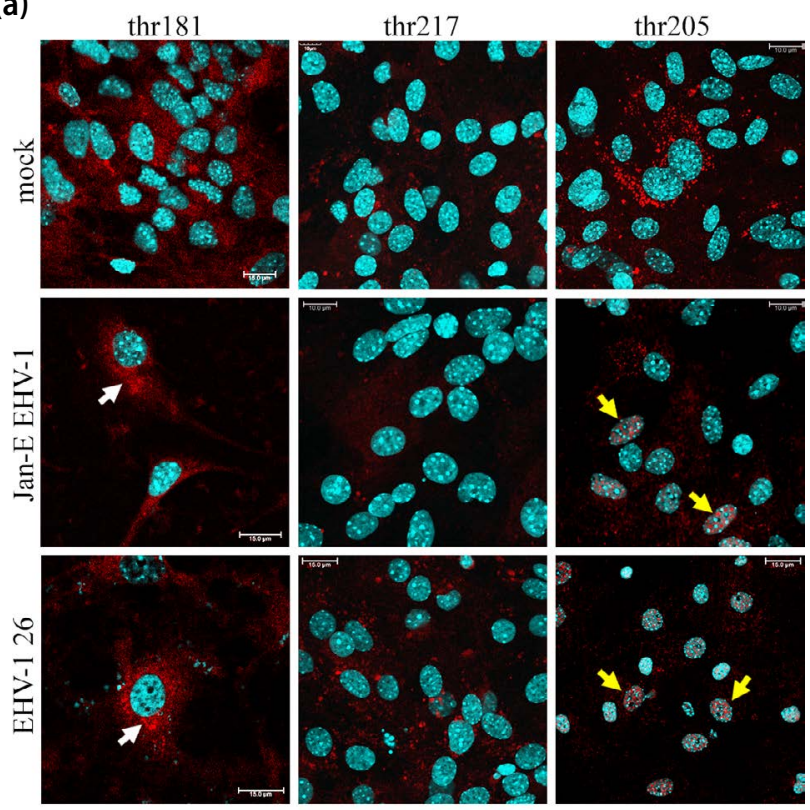

(b)
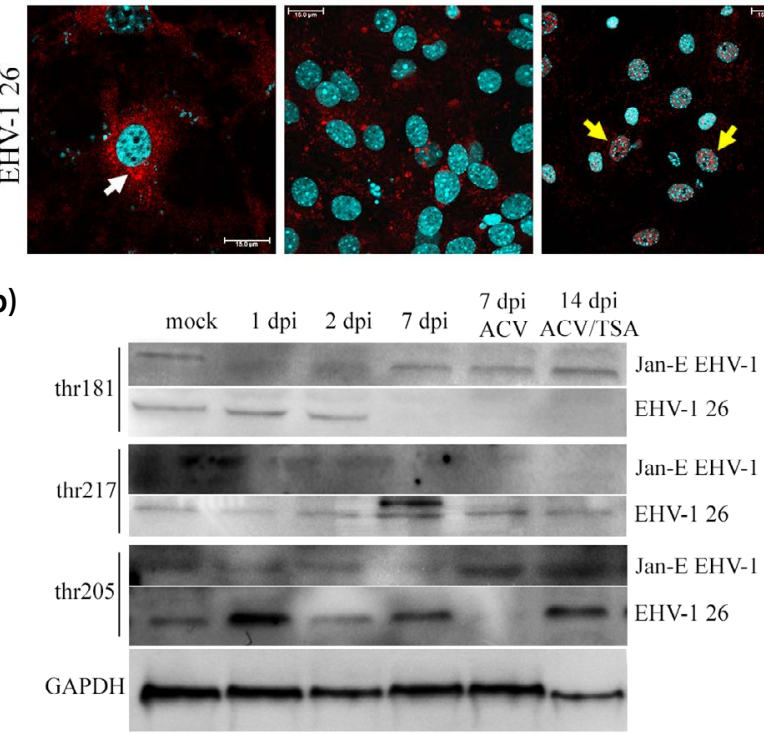

(c)

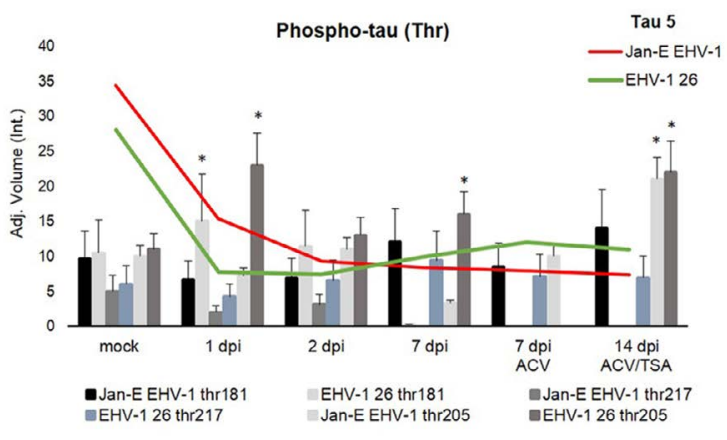

Fig. 3

Analysis of tau phosphorylation (thr181, thr217, thr205) expression

(a) Fluorescent images of mock- or EHV-1 infected primary murine neurons (1 dpi). Phospho-tau is shown in red and DNA in blue. White arrows indicate accumulation of phospho-tau thr181 around the nucleus. Yellow arrows indicate intranuclear accumulation of phospho-tau thr205. (b) Western blot detection of thr181, thr217 and thr205 in mock- and EHV-1-infected neurons. Acyclovir (ACV) was used to inhibit EHV-1 replication 7 dpi. Trichostatin A (TSA) was used to stimulate productive infection at 14 dpi. GAPDH represents a loading control. (c) Densitometric analysis of thr181, thr217 and thr205 protein levels (from three independent experiments) normalized to GAPDH level in mock-infected and EHV-1-infected neurons; results were statistically compared to mock infected control ( $\left.{ }^{*} \mathrm{p} \leq 0.05\right)$. Red (Jan-E EHV-1) and green (EHV-26 strain) lines present total Tau 5 level in EHV-1-infected neurons. 
still raises many questions but, to our knowledge, this is the first report indicating that EHV-1 infection alters the tau phosphorylation state in neurons.

Acknowledgments. Results were partially presented at the 10th International Virology Summit on July 02-04, 2018 at Vienna, Austria, in which JC's participation was supported by the Leading National Research Centre (KNOW) Scientific Consortium "Healthy Animal - Safe Food," decision of Ministry of Science and Higher Education No. 05-1/KNOW2/2015. This work was supported by grant No DEC-2017/01/X/NZ3/00435 (to JC) from the National Science Center in Cracow, Poland (https:// www. ncn.gov.pl/).

\section{References}

Alvarez G, Aldudo J, Alonso M, Santana S, Valdivieso F (2012): Herpes simplex virus type 1 induces nuclear accumulation of hyperphosphorylated Tau in neuronal cells. J. Neurosci. Res. 90, 1020-1029. https://doi.org/10.1002/ jnr.23003

Arendt T, Stieler JT, Holzer M (2016): Tau and taupathies. Brain Res. Bull. 126, 238-292. https://doi.org/10.1016/i.brainresbull.2016.08.018

Brunden KR, Ballatore C, Lee VMY, Smith A, Trojanowski JQ (2012): Brain-penetrant microtubule-stabilizing compounds as potential therapeutic agents for taupathies. Biochem. Soc. Trans. 40, 661-666. https://doi. org/10.1042/BST20120010

Cymerys J, Dzieciątkowski T, Słońska A, Bierła J, Tucholska A, Chmielewska A, Golke A, Bańbura MW (2010): Equine herpesvirus type 1 (EHV-1) replication in primary murine neurons culture. Pol. J.Vet. Sci.13, 701-708. https:// doi.org/10.2478/v10181-010-0022-3

Cymerys J, Słońska A, Brzezicka J, Tucholska A, Chmielewska A, Rola J, Malik P, Bańbura MW (2016): Replication kinetics of neuropathogenic and non-neuropathogenic equine herpesvirus type 1 (EHV-1) strains in primary murine neurons and ED cell line. Pol. J. Vet. Sci. 19, 777-784. https://doi.org/10.1515/pjvs-2016-0098

Cymerys J, Słońska A, Tucholska A, Golke A, Chmielewska A, Bańbura MW(2018): Influence of long-term equine her- pesvirus type 1 (EHV-1) infection on primary murine neurons - the possible effects of the multiple passages of EHV-1 on its neurovirulence. Folia Microbiol. 63,1-11. https://doi.org/10.1007/s12223-017-0528-5

Golke A, Cymerys J, Tucholska A, Chmielewska A, Słońska A, Chodkowski M, Serafińska I, Brzezicka J, Bańbura MW (2017): Acyclovir and trichostatin A modulate EHV-1 replication in murine neurons in vitro. Med. Wet. 73, 632-637. https://doi.org/10.21521/mw.5788

Himmelstein D, Ward SM, Lancia JK, Patterson KR, Binder LI (2012): Tau as a therapeutic target in neurodegenerative disease. Pharmacol. Terapeutic 136, 8-22. https:// doi.org/10.1016/j.pharmthera.2012.07.001

Hussey GS, Goehring LS, Lunn DP, Hussey SB, Huang T, Osterrieder N, Powell C, Hand J, Holz C, Slater J (2013): Experimental infection with equine herpesvirus type 1 (EHV-1) induces chorioretinal lesions. Vet. Res.44,118. https://doi.org/10.1186/1297-9716-44-118

Kristen H, Santana S, Sastre I, Recuero M, Bullido M, Aldudo J (2015): Herpes simplex virus type 2 infection induces AD-like neurodegeneration markers in human neuroblastoma cells. Neurobiol. Aging 36, 2737-2747. https:// doi.org/10.1016/j.neurobiolaging.2015.06.014

Laurent C, Buee L, Blum D (2018): Tau and neuroinflammation: What impact for Alzheimer's Disease and Taupaties? Biomed. J. 41, 21-33. https://doi.org/10.1016/j. bj.2018.01.003

Lin W, Wozniak MA, Cooper RJ, Wilcock GK, Itzhaki RF (2002): Herpesviruses In brain and Altheimer's disease. J. Path. 197, 395-402. https://doi.org/10.1002/path.1127

Liu Y, Li S (2015): A cell culture model of latent and lytic herpes simplex virus type 1 infection in spiral ganglion. ORL Otorhinolaryngol. Relat. Spec. 77, 141-149. https://doi. org/10.1159/000381679

Malik P, Pálfi V, Bálint A (2010): Development of a new primerprobe energy transfer method for the differentiation of neuropathogenic and non-neuropathogenic strains of equine herpesvirus-1.J.Virol. Met.169, 425-427. https:// doi.org/10.1016/i.jviromet.2010.08.007

Wozniak MA, Frost AL, Itzhaki RF (2009): Alzheimer's diseasespecific tau phosphorylation is induced by herpes simplex virus type 1. J. Alzheimers Dis. 16, 341-350. https://doi.org/10.3233/JAD-2009-0963 